\section{Endoscopic submucosal dissection for residual rectal polyps embedded in tissue scar: a "rescue therapy" to prevent surgical intervention?}

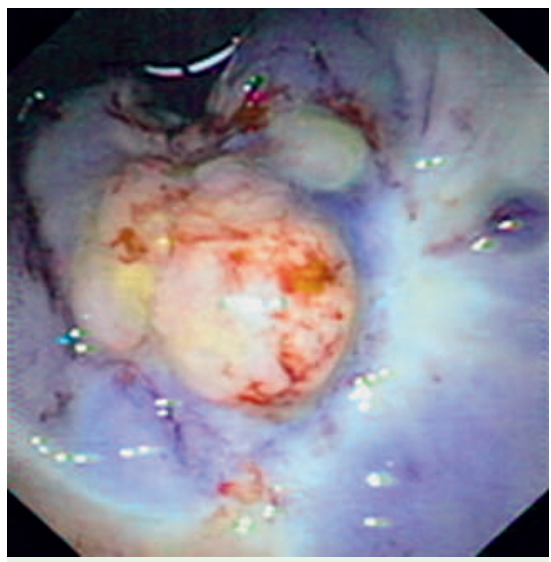

Fig. 1 Creation of a submucosal cushion. About $40 \mathrm{ml}$ of a mixed solution of hyaluronic acid preparation $(0.5 \%)$, saline, and diluted epinephrine $(1: 40000)$ was injected into the submucosal layer. "No lifting sign" was present.

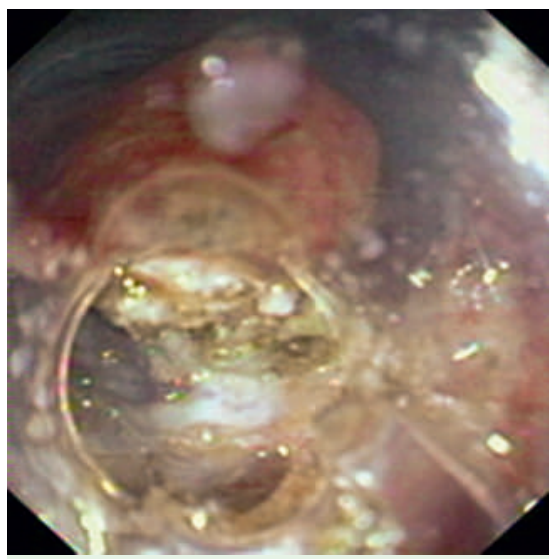

Fig. 4 Using a lateral movement and stressing the submucosal fibers using the transparent cap. Hemorrhage or visible vessels were treated by soft $60 \mathrm{~W}$ electrocoagulation using a coagulation forceps (Pentax SDB2422, Maeno-cho, Itabashi-ku, Tokyo, Japan) or by positioning metallic clips (Quick clip 2, HX-201UR135, Tokyo, Japan).

The authors report a case of a 48-year-old Caucasian woman with a $3.5 \mathrm{~cm}$ residual polyp embedded in tissue scar, as a result of three endoscopic piecemeal resection sessions of a $10 \mathrm{~cm}$ sessile polyp of the lower rectum, previously diagnosed from biopsy as tubulous-villous adenoma with high-grade dysplasia.

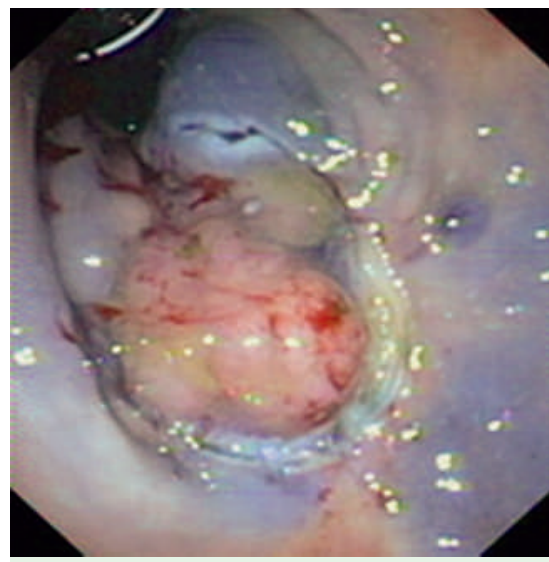

Fig. 2 Incision of the circumferential margins with standard needle knife in $120 \mathrm{~W}$ endocut mode (Erbe ICC 200, Tubingen, Germany).

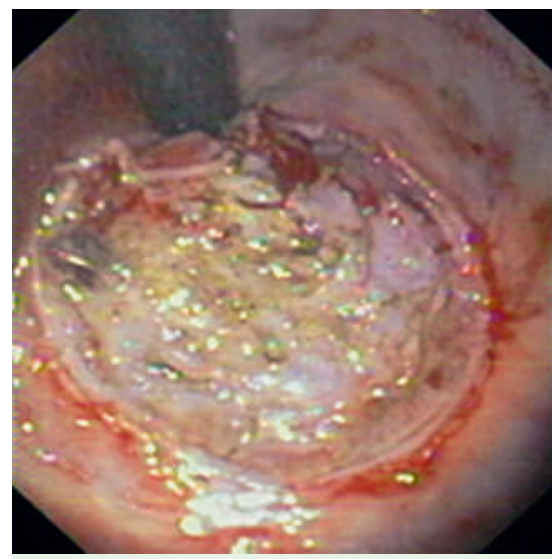

Fig. 5 Operatory field at the end of the procedure. The polyp was extracted and subsequently orientated on a rigid support.

The patient was then submitted to en bloc resection with endoscopic submucosal dissection (ESD) according to the technique of Yamamoto [1,2] ( Fig. 1-3). The preparation of the patient consisted of mechanical bowel cleansing with polyethylene glycol solution and 5 days of oral antibiotic therapy with ciprofloxacin (500 mg twice daily) and metronidazole (500 mg three times daily).

The procedure was carried out using a single-channel upper gastrointestinal endoscope with a water-jet system (Olympus

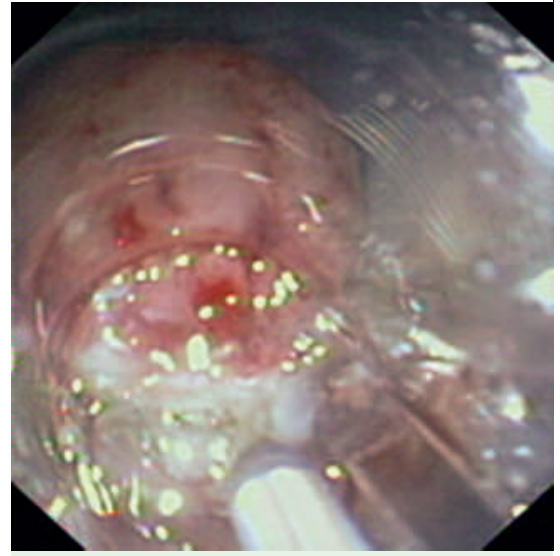

Fig. 3 Dissection of the submucosal layer with standard needle knife in $25 \mathrm{~W}$ forced mode. When the submucosal tissue was particularly hard due to scarring, the dissection was performed in $120 \mathrm{~W}$ endocut mode.

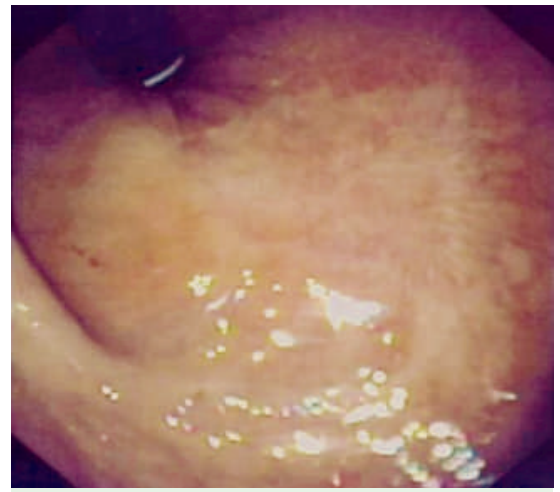

Fig. 6 The resected area 6 months after the procedure ("restitutio ad integrum").

GIF 1T-160, Tokyo, Japan). A transparent cap (ST-HOOD, DH 15GR, Fujinon, Saitama, Japan) was attached to the tip of the endoscope in order to apply tension to the submucosal connective fibers during dissection ( Fig.4-5). The procedure time was 2 hours. The postoperative course was uneventful and the patient was discharged 2 days after the procedure. The histological examination of the resected specimen described a residual adenomatous tissue with high-grade dysplasia; the excision margins were negative (R0 resection). The patient underwent control endoscopy 6 months later ( Fig.6), and multiple biopsies were 
taken of the resected area, which were negative at the histological examination. In cases of large polyps the standard of care is endoscopic piecemeal resection that, unfortunately, carries two disadvantages: the margins of resection may be difficult to evaluate by the pathologist, and in $14 \%-50 \%$ of cases at least one additional endoscopic session is required [3]. Notably, further endoscopic resection is often difficult as a result of fibrosis. These concerns are emphasized in large villous sessile rectal polyps because of their high potential for malignant transformation. In the reported case, the en bloc resection of a residual polyp, not amenable to standard endoscopic treatment (including endoscopic mucosal resection), was accomplished by ESD, avoiding a more invasive surgical procedure. As reported for residual/recurrence of early gastric cancer after endoscopic mucosal resection $[4,5]$, ESD can be proposed as an interesting endoscopic "rescue therapy" for residual rectal scar-embedded polyps.
Endoscopy_UCTN_Code_TTT_1AQ_2AD

\section{F. Azzolini, F. Biolchini, R. Sassatelli, L. Camellini, F. Decembrino, V. Iori, C. Tioli, G. Sereni, G. Bedogni}

Unit of Digestive and Biliopancreatic Endoscopy, Arcispedale Santa Maria

Nuova, Reggio Emilia, Italy

\section{References}

1 Gotoda T, Yamamoto H, Soetikno RM. Endoscopic submucosal dissection of early gastric cancer. J Gastroenterol 2006; 41: 929 942

2 Yamamoto H, Kita H. Endoscopic therapy of early gastric cancer. Best Pract Res Clin Gastroenterol 2005; 19: 909-926

3 Boix J, Lorenzo-Zuniga V, Moreno de Vega $V$ et al. Endoscopic removal of large sessile colorectal adenomas: is it safe and effective? Dig Dis Sci 2007; 52: 840-844

4 Oka S, Tanaka S, Kaneko I et al. Endoscopic submucosal dissection for residual/local recurrence of early gastric cancer after endoscopic mucosal resection. Endoscopy 2006 ; 38: $996-1000$

5 Fujishiro M, Goto O, Kakushima N et al. Endoscopic submucosal dissection of stomach neoplasms after unsuccessful endoscopic resection. Dig Liver Dis 2007; 39: 566-571
Bibliography

DOI $10.1055 / \mathrm{s}-2008-1077441$

Endoscopy 2008; 40: E222 - E223

(c) Georg Thieme Verlag KG Stuttgart · New York . ISSN 0013-726X

Corresponding author

\section{F. Azzolini, MD}

Unit of Digestive and Biliopancreatic Endoscopy Arcispedale Santa Maria Nuova

viale risorgimento 80

42200 Reggio Emilia

Italy

Fax: +39-0522-296129

francesco.azzolini@asmn.re.it 